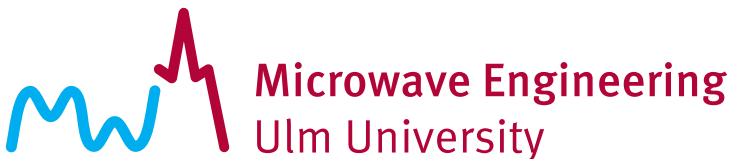

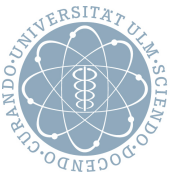

\section{Estimation and Cancellation of Interferences in Automotive Radar Signals}

\author{
Jonathan Bechter, Kushan Deb Biswas, and Christian Waldschmidt
}

(c) 2017 IEEE. Personal use of this material is permitted. Permission from IEEE must be obtained for all other uses, in any current or future media, including reprinting/republishing this material for advertising or promotional purposes, creating new collective works, for resale or redistribution to servers or lists, or reuse of any copyrighted component of this work in other works. 


\title{
Estimation and Cancellation of Interferences in Automotive Radar Signals
}

\author{
Jonathan Bechter, Kushan Deb Biswas, Christian Waldschmidt
}

\author{
Institute of Microwave Engineering \\ Ulm University, Germany \\ email: jonathan.bechter@uni-ulm.de
}

\begin{abstract}
Radar sensors become typical components for automotive driver assistance systems and autonomous driving. The widespread use of this sensor type leads to an increasing risk of unwanted interferences. Those interferences can severely degrade the detection performance and cause sensor blindness. To overcome this problem, this paper describes a method to estimate and remove interfering signals in data of chirp sequence modulated radars. The estimation is derived theoretically and applied on simulation and measurement data.
\end{abstract}

\section{Introduction}

Future generations of vehicles in the civil sector get equipped with driver assistance systems for safety and comfort functions. These systems rely on a variety of sensors, of which one typical kind is the radar sensor. Thus, the amount of radar sensors which operate at the same time in the same frequency range increases, leading to a high risk of unwanted interferences between them.

Investigations on the interference potential and effects between FMCW (frequency modulated continuous wave) radars showed that such interferences can severely decrease the detection capability of the sensors $[1,2]$. The most common interference scenario is an intersection of the frequency ramps transmitted by different sensors [3]. Such interference increases the receiver's noise floor and can lead to a sensor blindness. Especially targets with a low radar cross section, like pedestrians, cannot be detected reliably anymore due to this effect.

This paper addresses interferences between FMCW radars with short frequency ramps (chirp sequence radars). The generation of such interferences is described in Section 2. As a countermeasure we developed a method to remove the interference from the receive signal [4]. This work was carried on and an improved version of this method is derived in Section 3. Its effectivity is demonstrated with data from a chirp sequence modulated radar in Section 4 . The results are compared to a common interference countermeasure, the notch approach. 


\section{Automotive Radar Interference}

A chirp sequence radar transmits a series of linear frequency ramps (chirps) of the form

$$
s_{\mathrm{Tx}}(t) \propto \cos \left(2 \pi \int\left(f_{c}+\frac{B}{T_{c}} t\right) d t\right) .
$$

A single chirp has the duration $T_{c}$, the carrier frequency $f_{c}$, and the bandwidth $B$. The ramps are reflected from objects in the radar channel and return to the sensor after a time delay $\tau$. The transmit and receive signal are mixed and afterwards filtered with a low-pass filter (LPF) according to Fig. 1. The baseband signal of the $k$-th chirp is [5]

$$
s_{\mathrm{BB}}(t)=A_{\mathrm{BB}} \cos \left(2 \pi\left(\frac{2 f_{c} R}{c}+\frac{2 f_{c} v}{c} k T_{\mathrm{r} 2 \mathrm{r}}+\left(\frac{2 B R}{c T_{c}}+\frac{2 f_{c} v}{c}\right) t\right)\right) .
$$

$v$ and $R$ are velocity and distance of a target, $T_{\mathrm{r} 2 \mathrm{r}}$ is the time between two chirps, and $c$ is the speed of light. The signal has the amplitude $A_{\mathrm{BB}}$. The baseband signal is digitized with an analog to digital converter (ADC) and processed with a window function and a two-dimensional Fourier Transform to extract range and velocity. In this work, a Kaiser window with $\beta=5$ is applied.
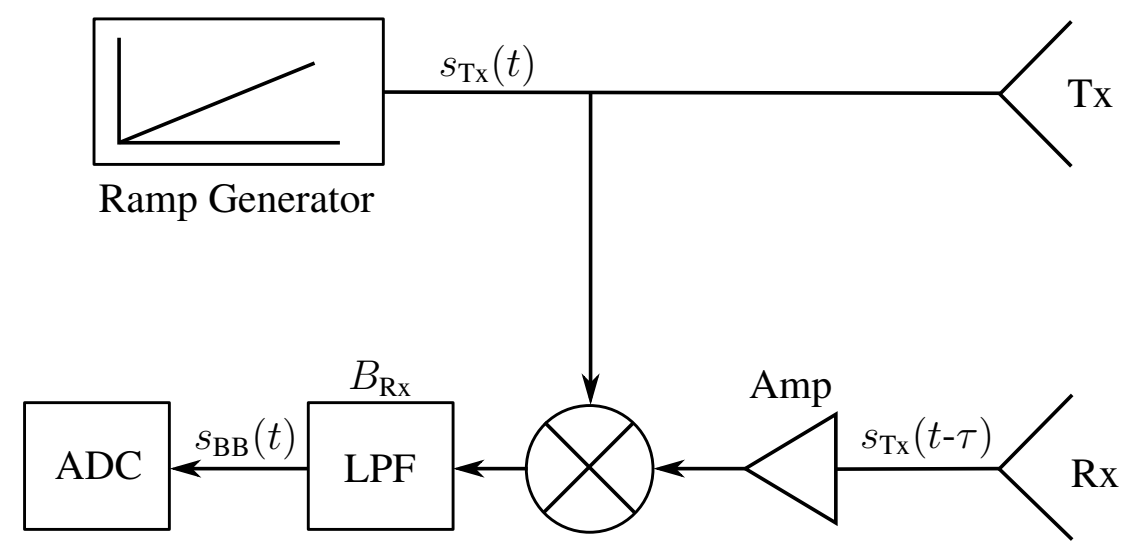

Figure 1: Simplified block diagram of a chirp sequence radar.

In Fig. 2a two sensors operate at the same time and their frequency ramps intersect. Interference occurs in the baseband signal of sensor 1 as long as the interfering signal falls into the receiver bandwidth $B_{\mathrm{Rx}}$ limited by the low-pass filter in Fig. 1 . The interference baseband signal is a time-limited frequency ramp with a high amplitude level. Its duration is [2]

$$
T_{\mathrm{Int}}=\frac{2 B_{\mathrm{Rx}}}{\frac{B_{\mathrm{Int}}}{T_{c, \text { Int }}}-\frac{B}{T_{c}}}
$$

$B_{\text {Int }}$ and $T_{c \text {,int }}$ thereby describe the bandwidth and ramp duration of the interfering radar sensor. The time-frequency behavior is determined by the slopes of the transmitted chirps [2,4]. The interference baseband signal is described generally as

$$
s_{\text {Int }}(t)=A_{\text {Int }} \cos \varphi_{\text {Int }}(t)
$$




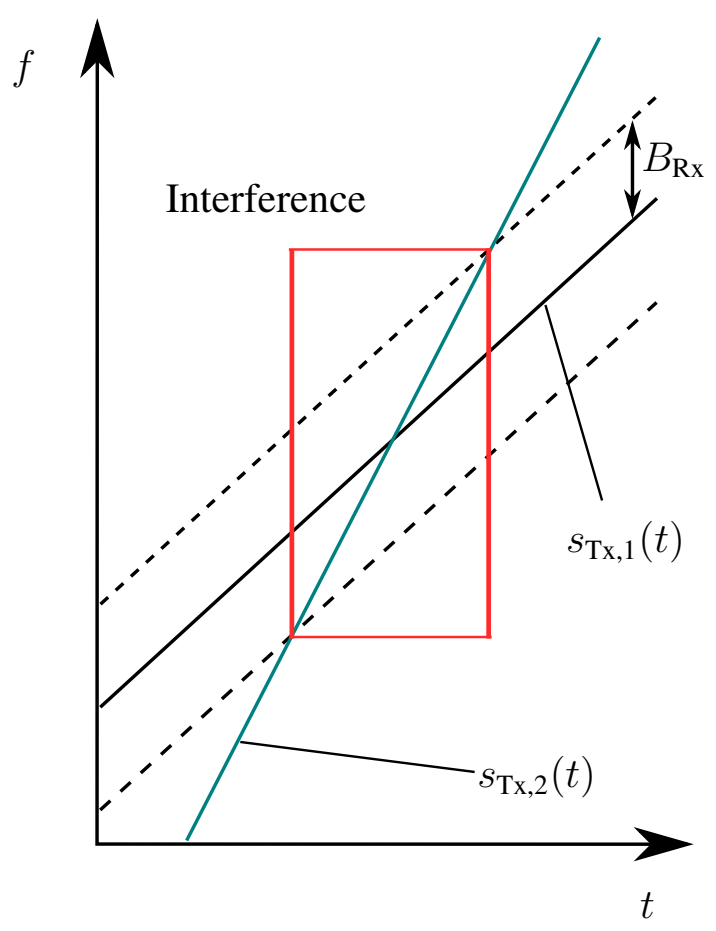

(a) The frequency ramps of two sensors intersect. This leads to unwanted interference.

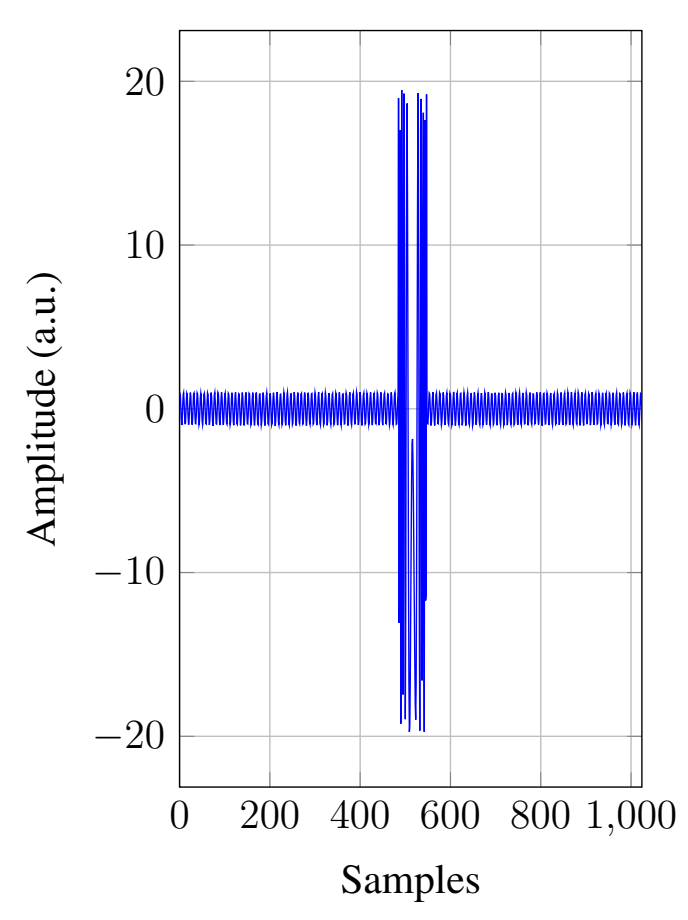

(b) Simulated baseband signal of sensor 1 with an ideal LPF in the receive path.

Figure 2: Two sensors transmit frequency ramps $s_{\mathrm{Tx}, 1}(t)$ and $s_{\mathrm{Tx}, 2}(t)$ and interference occurs. Its duration is limited by the receiver bandwidth $B_{\mathrm{Rx}}$. The baseband signal is affected by a short chirp with a high amplitude.

with

$$
\begin{aligned}
\varphi_{\mathrm{Int}}(t) & =a t^{2}+b t+c, \\
a & =\pi\left(\frac{B_{\mathrm{Int}}}{T_{c, \mathrm{Int}}}-\frac{B}{T_{\mathrm{c}}}\right) .
\end{aligned}
$$

The parameters $b$ and $c$ are determined by the point in time when the interference occurs and the zero phases of the signals, but they are not further specified here. Equation (5) shows that the phase of the interference signal follows the form of a parabola. For an interfering signal which completely intersects $B_{\mathrm{Rx}}$ as in Fig. 2a, the vertex of the parabola is the center of the interference duration. At this point, the frequency of the down converted interference signal is zero.

\section{Interference Signal Estimation}

In this section, phase and amplitude of the interfering signal are estimated. It is assumed that the amplitude of the interfering signal is much higher than the amplitudes of the desired signals (cp. [1]), so the signal part affected by interference can be detected with a power detec- 
tor [6]. It is assumed that the interfered receive signal $\underline{s}_{\mathrm{BB}, \text { Int }}(t)$ is complex and of the form

$$
\begin{aligned}
\underline{s}_{\mathrm{BB}, \mathrm{Int}}(t) & =\underline{s}_{\mathrm{BB}}(t)+\underline{s}_{\mathrm{Int}}(t) \\
\underline{s}_{\mathrm{BB}}(t) & =\sum_{n} A_{\mathrm{BB}, n}\left(\cos \varphi_{\mathrm{BB}, n}(t)+\mathrm{j} \sin \varphi_{\mathrm{BB}, n}(t)\right) \\
\underline{s}_{\mathrm{Int}}(t) & =A_{\mathrm{Int}}\left(\cos \varphi_{\mathrm{Int}}(t)+\mathrm{j} \sin \varphi_{\mathrm{Int}}(t)\right)
\end{aligned}
$$

$\underline{s}_{\mathrm{BB}}(t)$ is the complex, interference-free receive signal for $n$ targets. The time derivative of the complex signal is

$$
\frac{d}{d t} \underline{s}_{\mathrm{BB}, \mathrm{Int}}(t)=\frac{d}{d t} \underline{s}_{\mathrm{BB}}(t)+A_{\mathrm{Int}}\left(\mathrm{j} \cos \varphi_{\mathrm{Int}}(t)-\sin \varphi_{\mathrm{Int}}(t)\right) \frac{d}{d t} \varphi_{\text {Int }}(t) .
$$

The derivative of the mono-frequent baseband signals is

$$
\frac{d}{d t} \underline{s}_{\mathrm{BB}}(t)=\sum_{n} A_{\mathrm{BB}, n}^{\prime}\left(\mathrm{j} \cos \varphi_{\mathrm{BB}, n}(t)-\sin \varphi_{\mathrm{BB}, n}(t)\right) .
$$

The ratio of real to imaginary part in (10) is

$$
\begin{gathered}
\frac{\operatorname{Re}\left(\frac{d}{d t} \underline{s}_{\mathrm{BB}}(t)\right)}{\operatorname{Im}\left(\frac{d}{d t} \underline{s}_{\mathrm{BB}}(t)\right)}=\frac{-A_{\mathrm{Int}} \sin \varphi_{\mathrm{Int}}(t) \frac{d}{d t} \varphi_{\mathrm{Int}}(t)-\sum_{n} A_{\mathrm{BB}, n}^{\prime} \sin \varphi_{\mathrm{BB}, n}(t)}{A_{\mathrm{Int}} \cos \varphi_{\mathrm{Int}}(t) \frac{d}{d t} \varphi_{\mathrm{Int}}(t)+\sum_{n} A_{\mathrm{BB}, n}^{\prime} \cos \varphi_{\mathrm{BB}, n}(t)} \\
=\frac{-\sin \varphi_{\mathrm{Int}}(t)-x \sum_{n} A_{\mathrm{BB}, n}^{\prime} \sin \varphi_{\mathrm{BB}, n}(t)}{\cos \varphi_{\mathrm{Int}}(t)+x \sum_{n} A_{\mathrm{BB}, n}^{\prime} \cos \varphi_{\mathrm{BB}, n}(t)} \approx \frac{-\sin \varphi_{\mathrm{Int}}(t)}{\cos \varphi_{\mathrm{Int}}(t)},
\end{gathered}
$$

with

$$
x=\frac{1}{A_{\text {Int }} \frac{d}{d t} \varphi_{\text {Int }}(t)} .
$$

Equation (14) approximates that

$$
x \sum_{n} A_{\mathrm{BB}, n}^{\prime} \sin \varphi_{\mathrm{BB}, n}(t) \approx x \sum_{n} A_{\mathrm{BB}, n}^{\prime} \cos \varphi_{\mathrm{BB}, n}(t) \approx 0 .
$$

It was shown in simulations that the derivative of the signal is dominated by the interference component [4], so the above approximation is valid. The only exception is the time period when $d \varphi_{\text {Int }}(t) / d t \approx 0$. This inaccuracy is visible at the vertex of the parabola in the later shown Fig $6 a$.

It can be seen that (14) holds an estimation $\hat{\varphi}_{\text {Int }}(t)$ of the phase of the interference component:

$$
\frac{\operatorname{Re}\left(\frac{d}{d t} \underline{s}_{\mathrm{BB}}(t)\right)}{\operatorname{Im}\left(\frac{d}{d t} \underline{s}_{\mathrm{BB}}(t)\right)}=-\tan \hat{\varphi}_{\mathrm{Int}}(t) .
$$

In the next step, the amplitude $A_{\text {Int }}$ is determined. Therefore, an initial real-valued interference signal is calculated as

$$
\hat{s}_{\text {Int }}(t)=\hat{A}_{\text {Int }} \cos \hat{\varphi}_{\text {Int }}(t),
$$


with an arbitrary value $\hat{A}_{\text {Int }}$. With a scaling factor $\alpha$, the real-valued interference-free signal is estimated as

$$
\hat{s}_{\mathrm{BB}}(t)=s_{\mathrm{BB}, \mathrm{Int}}(t)-\alpha \hat{s}_{\mathrm{Int}}(t)
$$

or in a discrete notation as

$$
\hat{s}_{\mathrm{BB}}\left(k T_{s}\right)=s_{\mathrm{BB}, \mathrm{Int}}\left(k T_{s}\right)-\alpha \hat{s}_{\mathrm{Int}}\left(k T_{s}\right),
$$

with the sampling time $T_{s}$. The signal power is then

$$
P^{\prime}(\alpha)=\frac{1}{M} \sum_{k}\left(s_{\mathrm{BB}, \text { Int }}\left(k T_{s}\right)-\alpha \hat{s}_{\text {Int }}\left(k T_{s}\right)\right)^{2} .
$$

There, $k$ accesses all interfered samples and $M$ is their total number. The power of the interference-free signal is called $P$. The difference of these powers is described with a function

$$
g(\alpha)=P^{\prime}(\alpha)-P \geq 0 .
$$

For an optimum value of $\alpha$, the interference is removed completely from the signal and the function reaches a minimum. This leads to

$$
\begin{array}{r}
\frac{d g(\alpha)}{d \alpha}=\frac{d}{d \alpha} P^{\prime}(\alpha) \stackrel{!}{=} 0 \\
\frac{1}{M} \sum_{k} 2 \cdot\left(s_{\mathrm{BB}, \text { Int }}\left(k T_{s}\right)-\alpha \hat{s}_{\text {Int }}\left(k T_{s}\right)\right) \cdot\left(-\hat{s}_{\text {Int }}\left(k T_{s}\right)\right)=0
\end{array}
$$

and finally

$$
\alpha=\frac{\sum_{k} \hat{s}_{\text {Int }}\left(k T_{s}\right) s_{\mathrm{BB}, \mathrm{Int}}\left(k T_{s}\right)}{\sum_{k} \hat{s}_{\text {Int }}\left(k T_{s}\right)^{2}} .
$$

After $\alpha$ is determined with (25), the interference is removed completely with (19).

Equations (7) to (17) assumed complex receive signals, which can be achieved with an IQ receiver. However, an IQ receiver is typically not required for chirp sequence modulated radars. Thus, the imaginary signal part required in (9) is calculated with a Hilbert Transform. The simulation of an interfered signal in Fig. 3 compares the imaginary part generated with an IQ receiver and the imaginary part calculated with the Hilbert Transform of the corresponding real valued signal. Half of the interfered signal part fits very well, but the other half is phase shifted by $\pi$. The phase error occurs after the phase $\varphi_{\text {Int }}(t)$ reaches the vertex of the parabola described in (5). At this point, the frequency of the interfering signal shifts from positive to negative values or vice versa. The Hilbert Transform cannot describe this behavior, as it does not provide the additional information generated with an IQ receiver.

It is also possible that the observed phase shift of $\pi$ occurs before the vertex is reached. Anyway, the issue is solved by inverting the first half of the interfered signal. The imaginary part created this way is then correct or multiplied by a factor -1 . In the second case, the negative sign is carried on to (17). Due to the tangent inverse, it leads to a phase error of $\pi$ in $\varphi_{\text {Int }}(t)$, which is again a factor -1 . When $\alpha$ is calculated with (25), this error is corrected automatically. Thus, the Hilbert Transform can be used when an IQ receiver is not available. 


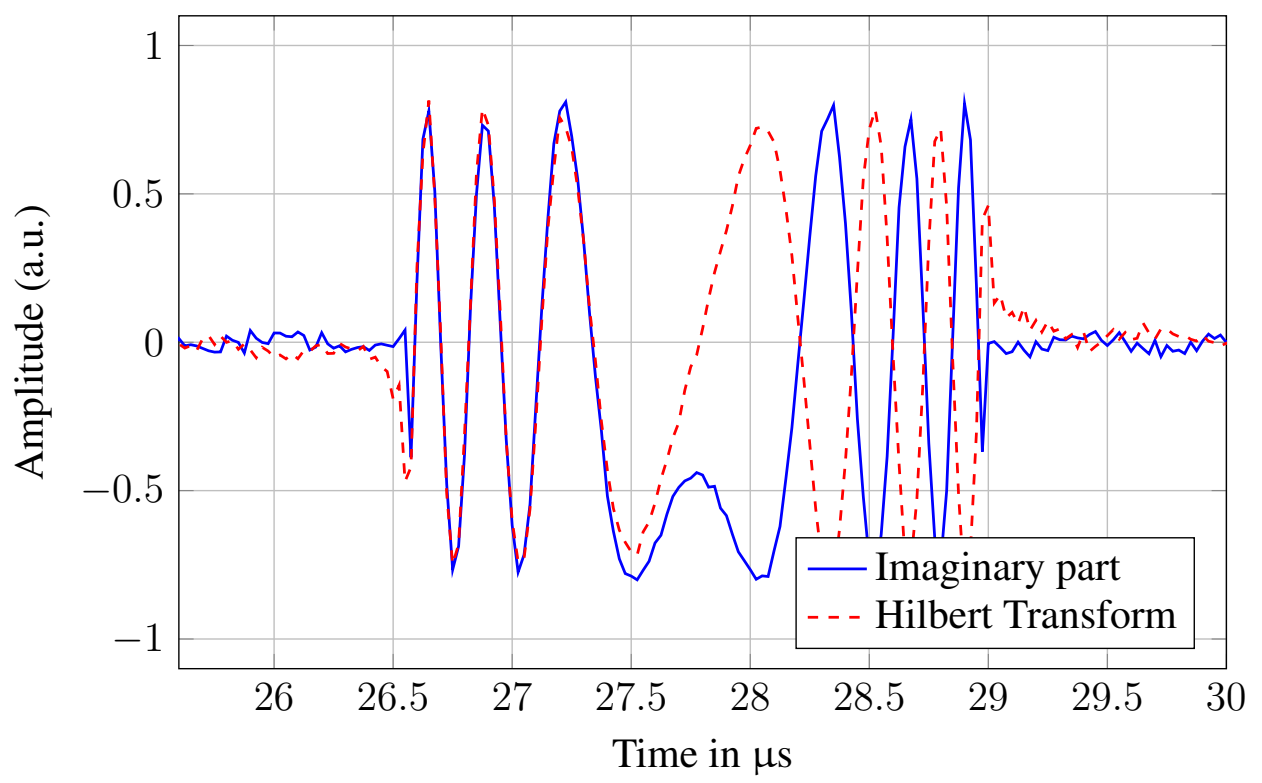

Figure 3: Simulative comparison of the imaginary part generated by an IQ receiver and the result of a Hilbert Transform applied on the data in Fig. $2 b$.

\section{Evaluation of the Method}

In this section, the described method is verified with simulation and measurement data. In the first part, the simulated signal in Fig. $2 b$ is repaired. This is done with a complex baseband signal, so the availability of an IQ receiver is assumed. The signal part affected by interference is shown in Fig. 4a after the algorithm is applied, compared to the same signal without interference from another sensor. The corresponding spectra in Fig. $4 \mathrm{~b}$ show, that the interference cannot be removed completely. The maximum gain in SNIR (signal to noise and interference ratio) that could be achieved in simulations is limited to around $26 \mathrm{~dB}$. Further simulations showed that the main limitation of this gain is the derivative required for (17). This derivative is approximated with the difference quotient, what leads to limited accuracy.

In the next step, the method is applied on measurement data. Therefore, two radar sensors are placed in a distance of $26 \mathrm{~m}$. The sensors operate in the $77 \mathrm{GHz}$ band and use chirp sequence modulations. The interfered sensor is not equipped with an IQ mixer, so the Hilbert Transform has to be applied. The interference duration is adjusted according to (3).

Fig. 5 shows the measurement of an interfered frequency ramp affected by a long interference period. However, the level of the interfering signal is not constant over time. The non-ideal receive path (especially the low-pass filter) has a frequency-dependent amplification while the interference baseband signal is a frequency ramp, thus $A_{\text {Int }}=A_{\text {Int }}(t)$. As (14) is independent of this amplitude, the estimation of $\varphi_{\text {Int }}(t)$ is still correct. The phase estimation of the signal in Fig. 5 is shown in Fig. 6a. From this phase, the frequency $f(t)$ is determined, see Fig. 6b. Note that the cut-off frequency of the low-pass filter in the receive path is $4 \mathrm{MHz}$. The amplitudefrequency dependency of the receive path is determined in a calibration measurement, so the 


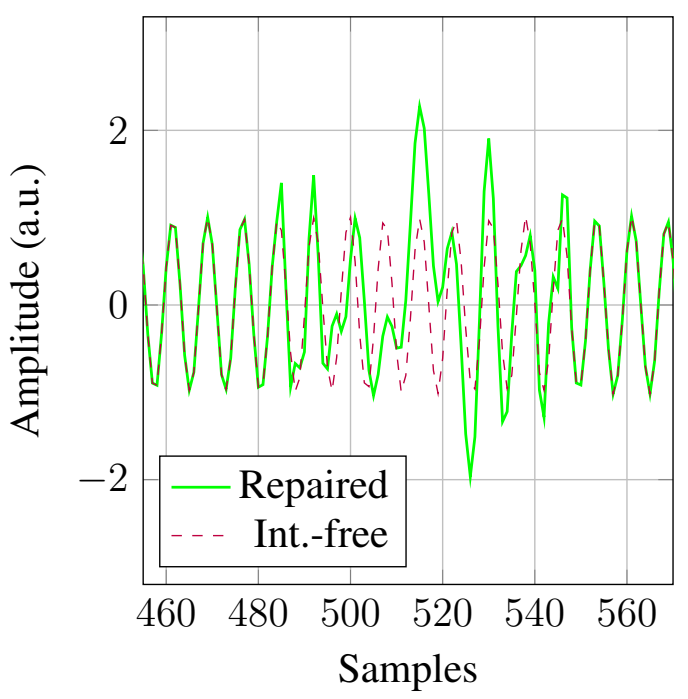

(a) Repaired and interference-free time signals corresponding to Fig. $2 \mathrm{~b}$.

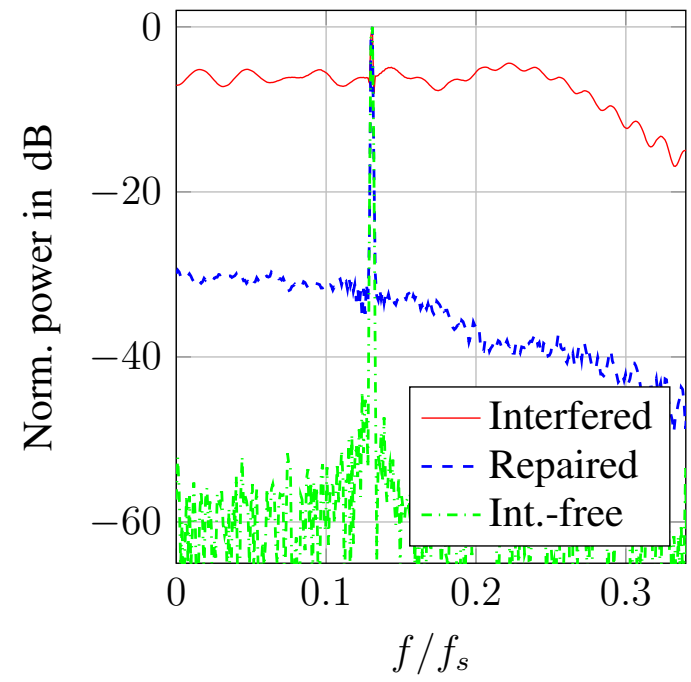

(b) Comparison of the spectra of the repaired, interfered, and interference-free signal.

Figure 4: Reconstruction of the simulated signal in Fig. 2b.

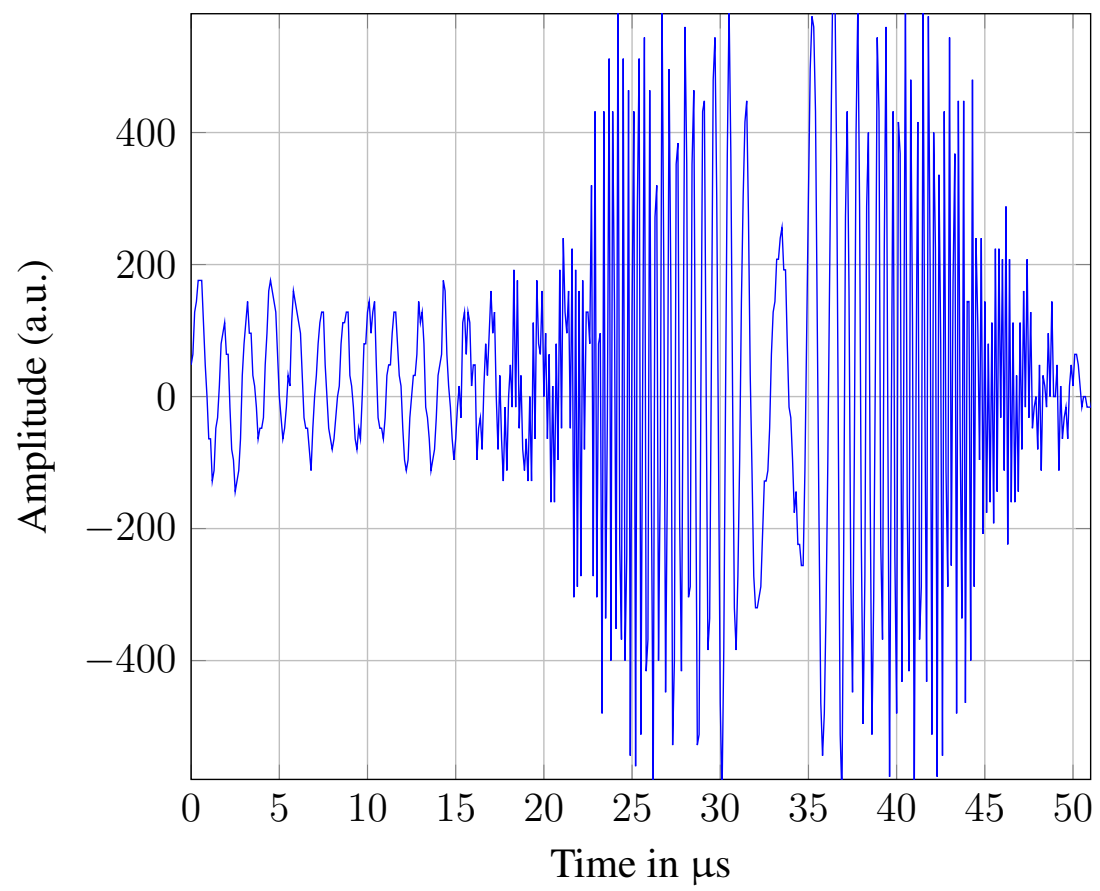

Figure 5: Measurement of an interfered time domain baseband signal with 235 of 512 samples affected by interference.

amplitude-over-time behavior can be modeled. It is taken into account for the interference reconstruction in (18).

The result of the interference reconstruction is depicted in Fig. 7a, the repaired time domain signal is shown in Fig. 7b. The frequency spectra of the original interfered signal and the repaired 


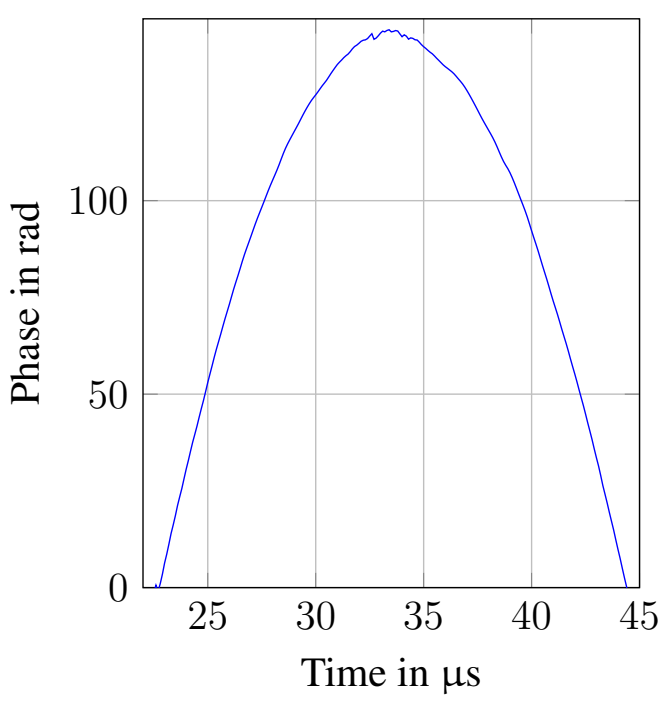

(a) The estimation of $\varphi_{\text {Int }}(t)$ is a parabola.

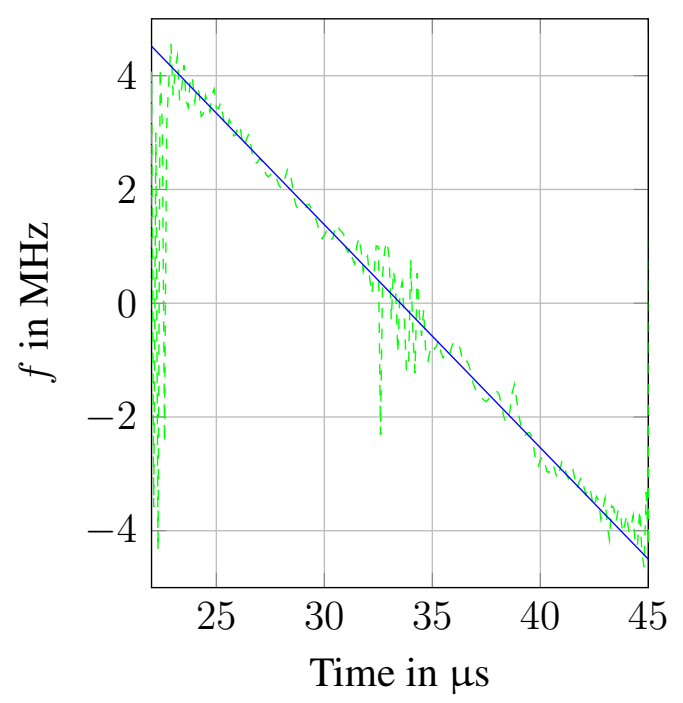

(b) Its derivative holds the frequency of $s_{\text {Int }}(t)$, optimized with a least-squares fit.

Figure 6: Estimation of phase and frequency of the long interference signal in Fig. 5.

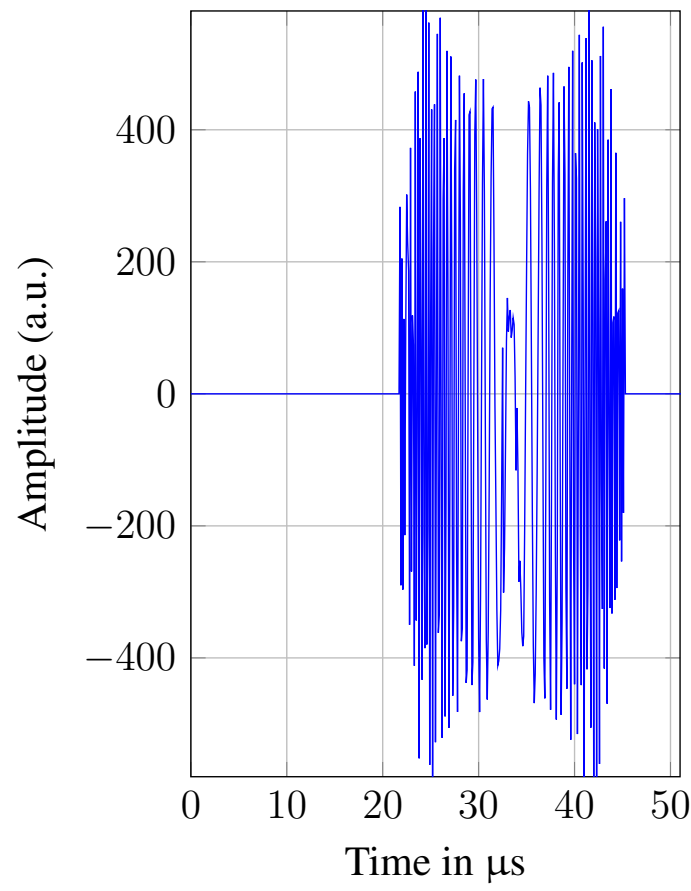

(a) Reconstructed interference component.

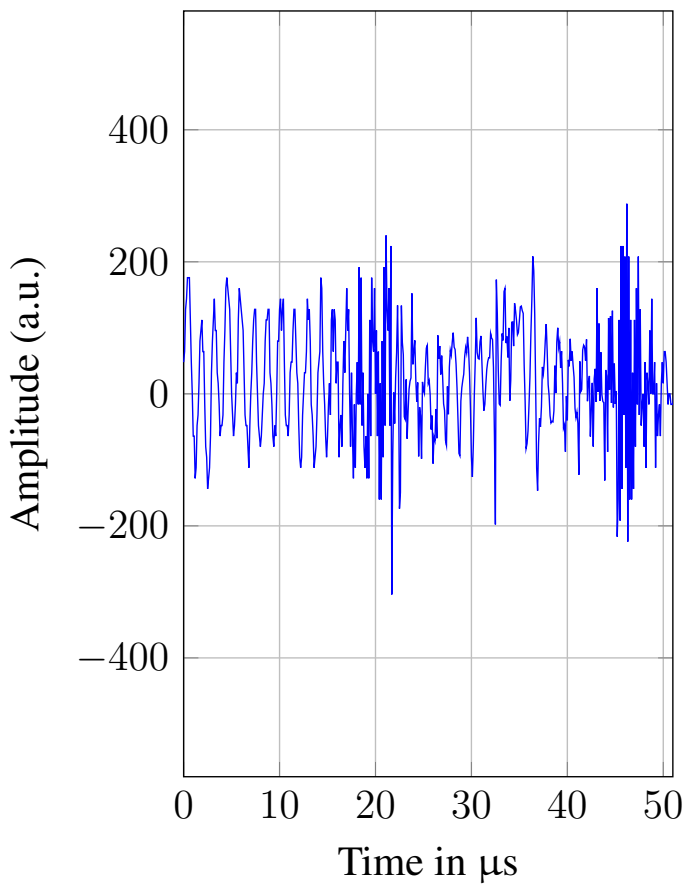

(b) Repaired time domain signal.

Figure 7: Estimation and cancellation of the interference signal in Fig. 5.

signal are compared in Fig. 8. Before the signal repair is applied, the interference-induced noise floor is only $9 \mathrm{~dB}$ below the target level. After repairing the signal, the interference level drops significantly and the target SNIR is $21.9 \mathrm{~dB}$. 


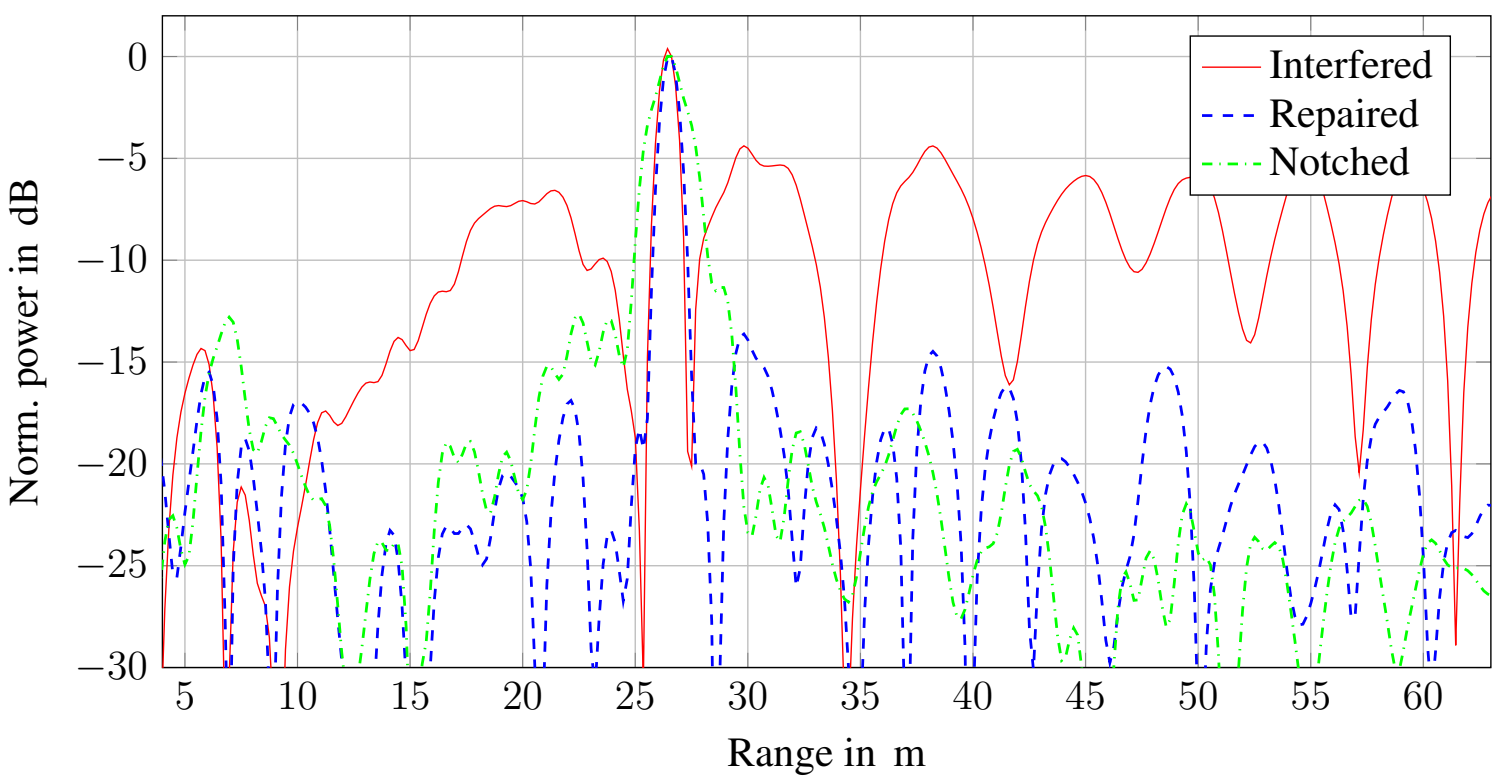

Figure 8: Frequency spectra normalized to the target at $26 \mathrm{~m}$.

The figure also shows what happens if the interfered samples of the time signal are simply notched instead of using the presented approach. Notching interfered samples is described as

$$
s_{\mathrm{BB}, \text { notched }}(t)=s_{\mathrm{BB}}(t) \cdot\left(\operatorname{rect}\left(\frac{t}{T_{c}}\right)-\operatorname{rect}\left(\frac{t-\tau}{T_{\mathrm{Int}}}\right)\right) .
$$

The first rectangle represents the total measurement time. The variable $\tau$ shifts the second rectangle to the interference period, removing this signal part. In the spectrum, notching convolves an additional si-function with all targets. Thereby it reduces their power levels and introduces additional artifacts. For the long interference duration, this effect is clearly visible. In this case, the sensor resolution is severely reduced, see the corresponding wide peak in Fig. 8. Still, notching raises the $\mathrm{SNR}$ to $19.4 \mathrm{~dB}$.

\section{Discussion and Conclusion}

The paper describes a method for the estimation of phase, frequency, and amplitude of interfering signals for chirp sequence modulated automotive radars. The estimated interference is removed from the receive signal to achieve interference-free operation. This method is especially effective for long interference durations with high power, as these circumstances improve the estimation accuracy. However, when applied on data with short interference durations, the method will not be the best choice. In case of short interference durations, the disadvantages of a simple notching approach are neglectable with much lower computation effort.

For longer interference durations the proposed method offers the advantage that the full signal information can be preserved compared to the notching of interfered samples. There is no loss in resolution and no additional artifacts are created in the spectrum (cp. Fig. 8). Simulations 
showed that a gain in SNR of up to $26 \mathrm{~dB}$ can be achieved. In measurements, an SNR improvement of $12.9 \mathrm{~dB}$ was possible, clearly outperforming the notching of interfered samples.

\section{References}

[1] G. M. Brooker, "Mutual Interference of Millimeter-Wave Radar Systems," IEEE Transactions on Electromagnetic Compatibility, vol. 49, no. 1, pp. 170-181, Feb. 2007.

[2] T. Schipper, M. Harter, T. Mahler, O. Kern, and T. Zwick, "Discussion of the operating range of frequency modulated radars in the presence of interference," International Journal of Microwave and Wireless Technologies, vol. 6, pp. 371-378, June 2014.

[3] D. Oprisan and H. Rohling, "Analysis of Mutual Interference between Automotive Radar Systems," in International Radar Symposium (IRS), 2005, Berlin.

[4] J. Bechter and C. Waldschmidt, "Automotive Radar Interference Mitigation by Reconstruction and Cancellation of Interference Component," in IEEE MTT-S International Conference on Microwaves for Intelligent Mobility (ICMIM), April 2015, pp. 1-4.

[5] V. Winkler, "Range Doppler Detection for automotive FMCW Radars," in European Microwave Conference, Oct. 2007, pp. 1445-1448.

[6] C. Fischer, M. Barjenbruch, H.-L. Blöcher, and W. Menzel, "Detection of Pedestrians in Road Environments with Mutual Interference," in 14th International Radar Symposium (IRS), vol. 2, June 2013, pp. 746-751. 